\title{
MELHORAMENTO GENÉTICO DA CANA-DE-AÇÚCAR: VI. ENSAIOS DE CLONES PROVENIENTES DE HIBRIDAÇÕES REALIZADAS EM 1974, 1977, 1978 E 1979, AVALIADOS NA REGIÃo DE PIRACICABA (SP) $\left(^{1}\right)$
}

\author{
ANTONIO PEREIRA DE CAMARGO $\left({ }^{2}\right)$, VIRGINIO BOVI ( $\left.{ }^{3}\right)$, RAPHAEL ALVAREZ $\left({ }^{3}\right)$, \\ GENTIL GODOY JUNIOR ( $)$, CELSO VALDEVINO POMMER ( $\left.{ }^{3}\right)$, MARCOS GUIMARÃES \\ DE ANDRADE LANDELL $\left({ }^{3}\right)$, LÉO ZIMBACK $\left({ }^{3}\right)$, TOSHIO IGUE ( $\left(^{5}\right)$ \\ e MARIA TERESA B. RAMOS DA SILVA $(9)$
}

\begin{abstract}
RESUMO
Testou-se uma série de clones obtidos de cruzamentos realizados em 1974, 1977, 1978 e 1979 no Instituto Agronómico, em dois locais na região de Piracicaba (SP). Os clones foram comparados com as testemunhas 'SP70-1143', 'LAC64-257', 'NA56-79' e 'CB41-76', em ensaios em blocos ao acaso, avaliando-se os caracteres agroindustriais na média de três cortes. Foram escolhidos para cultivo, na regiāo, o IAC78-23, por sua elevada produção de cana e açúcar, indicado para meio e fím đe safra, e o LAC79-1011, como material precoce, de produção média e alto teor de açúcar. Para colheita em meio de safra em solos férteis, foram escolhidos os seguintes clones de produçāo média e alto teor de açúcar: IAC77-186, LAC78-54, LAC78-90 e LAC79-1159.
\end{abstract}

Termos de indexação: cana-de-açúcar, Saccharum spp., melhoramento genético vegetal, teor de açúcar, hibridação e clones.

\section{ABSTRACT \\ SUGARCANE BREEDING: VI. EVALUATION OF CLONES OBTAINED IN 1974, 1977, 1978 AND 1979 HYBRIDIZATIONS IN PIRACICABA REGION, STATE OF SÃO PAULO, BRAZIL}

Sugarcane clones obtained in crosses made in 1974, 1977, 1978 and 1979, were tested in two locations in the Piracicaba region. The clones and commercial varieties SP70-1143, IAC64-257, NA56-79 and CB41-76 were evaluated for agricultural and industrial traits in an average of three harvests. The clone IAC78-23 was obtained for cultivation in the region with high cane and sugar yields and adequate for use in middle and late harvest, and the IAC79-1011 with medium yield and high sugar content, indicated for inicial harvest. The clones IAC77-186, IAC78-54, LAC78-90 and IAC79-1149 showed medium yield and high sugar content, indicated for middle harvest in soils with high fertility.

Index terms: sugarcane, Saccharum spp., breeding, yield, sugar content, crosses and clones.

(1) Trabalho recebido para publicação em 29 de janeiro e aceito em 2 de setembro de 1992.

$\left({ }^{2}\right)$ Estação Experimental de Piracicaba, IAC, Caixa Postal 28, 13400-970 Piracicaba (SP).

(3) Seçāo de Cana-de-Açucar, IAC, Caixa Postal 28, 13001-970 Campinas (SP).

() Estação Experimental de Ubatuba, IAC.

(5) Seção de Técnica Experimental e Cálculo, IAC.

(6) Seção de Fitoquímica, IAC. 


\section{INTRODUÇÃO}

O melhoramento genético convencional de cana-de-açúcar é trabalhoso e demorado. Desde a obtenção de sementes pela hibridação através da qual se almeja ampliar a variabilidade genética, passando pelas sucessivas seleções nas quais se procura isolar o fenótipo desejado e, finalmente, a experimentaçāo de campo em vários locais que possibilita a indicação de novos cultivares, transcorrem cerca de dez anos, apesar de se tratar de espécie propagada vegetativamente. Quando se dispõe de um programa contínuo de trabalho, anualmente podem surgir novas variedades, como vem acontecendo há mais de quarenta anos (Aguirre Jr., 1936; Alvarez et al., 1981, 1983, 1984, 1986, 1987a,b; Segalla \& Alvarez, 1964; Segalla et al., 1980, 1982; Campana et al., 1990).

A contínua busca de novas variedades é determinada pelo período relativamente curto de utilização das variedades comerciais de cana-de-açúcar, na maioria dos casos em função de doenças (Brieger, 1978) e da necessidade de obtenção de materiais superiores aos comerciais, visando aumentar os rendimentos agroindustriais de maneira econômica.

Este trabalho teve por objetivo estudar o comportamento agroindustrial de alguns clones selecionados a partir de cruzamentos efetuados em 1974, 1977, 1978 e 1979, em experimentos na regiāo de Piracicaba (SP), de 1987 a 1990.

\section{MATERIAL E METODOS}

O material utilizado neste trabalho originou-se de seleção a partir de cruzamentos realizados na Estação Experimental de Ubatuba, pela Seção de Cana-de-Açúcar, em 1974 e em 1977-79 na Estação Experimental de Camamu (BA), por deferimento da Cooperativa Central dos Produtores de Açúcar e Álcool (COPERSUCAR).

Foram instalados quatro experimentos, a saber: dois na Usina Costa Pinto, em Piracicaba, em solo podzólico vermelho, distrófico, e dois na Usina Santa Bárbara, em Santa Bárbara d'Oeste, em latossolo roxo distrófico. Os ensaios foram semelhantes nos dois locais, sendo um com clones das séries 1974 e 1977 e o outro com clones das séries 1978 e 1979.

No primeiro ensaio, empregou-se o delineamento de blocos ao acaso com cinco repetições, comparando-se nove clones com três variedades comerciais. No segundo, empregaram-se também cinco repetiçōes, mas com onze clones comparados com quatro variedades. Os clones do primeiro ensaio e seus respectivos parentais foram os seguintes:
Clones

IAC74-107

IAC74-339

IAC74-353

IAC77-36

IAC77-51

IAC77-153

IAC77-165

IAC77-173

IAC77-186

\section{Parentais}

$\begin{array}{lll}\text { CP48-103 } & \mathbf{x} & \left.?{ }^{7}\right) \\ \text { CP61-37 } & \mathrm{x} & \mathrm{PL}^{8}\left(^{8}\right) \\ \text { CP61-37 } & \mathrm{x} & \mathrm{PL} \\ \text { LAC58-480 } & \mathrm{x} & \text { LAC48-65 } \\ \text { IAC58-480 } & \mathrm{x} & \text { NA56-79 } \\ \text { Trojan } & \mathrm{x} & \text { Co } 775 \\ \text { IAC58-480 } & \mathrm{x} & ? \\ \text { IAC58-480 } & \mathrm{x} & ? \\ \text { IAC58-480 } & \mathrm{x} & ?\end{array}$

Os cultivares utilizados como testemunhas foram NA56-79, SP70-1143 e CB41-76, que, juntos, representavam $58 \%$ da área plantada com cana-de-açúcar do Estado de São Paulo (Machado Jr. et al., 1989). No segundo ensaio os clones foram:

\section{Clones}

IAC79-9

IAC78-12

LAC78-14

IAC78-20

IAC78-23

IAC78-54

IAC78-90

IAC79-1003

IAC79-1011

IAC79-1015

LAC79-1159

\section{Parentais}

$\begin{array}{lll}\text { CB41-76 } & \mathrm{x} & ? \\ \text { CB41-76 } & \mathrm{x} & ? \\ \mathrm{CB} 41-76 & \mathrm{x} & ? \\ \text { CP63-588 } & \mathrm{x} & ? \\ \text { IAC48-65 } & \mathrm{x} & ? \\ \mathrm{CP44-101} & \mathrm{x} & ? \\ \text { H 65-606 } & \mathrm{x} & ? \\ \text { IAC58-480 } & \mathrm{x} & \text { Co } 331 \\ \text { IAC52-150 } & \mathrm{x} & \mathrm{CP48-103} \\ \text { IAC52-150 } & \mathrm{x} & \mathrm{CP48-103} \\ \text { IAC57-219 } & \mathrm{x} & ?\end{array}$

As variedades testemunhas foram as mesmas do primeiro ensaio, acrescidas da 'LAC64-257'. As parcelas constituíram-se de três linhas de $8 \mathrm{~m}$, espaçadas de 1,40 m, com área útil de $33,6 \mathrm{~m}$. A adubação utilizada em cana-planta foi de $25 \mathrm{~kg}$ de $\mathrm{N}$, $120 \mathrm{~kg}$ de $\mathrm{P}_{2} \mathrm{O}_{5}$ e $120 \mathrm{~kg}$ de $\mathrm{K}_{2} \mathrm{O}$ no plantio e $60 \mathrm{~kg}$ de $\mathrm{N}$ e $80 \mathrm{~kg}$ de $\mathrm{K}_{2} \mathrm{O}$ em cobertura por hectare.

(7) Mistura de pólen de vários parentais masculinos.

( ) Polinização livre no campo. 
O plantio foi feito em março de 1987, procedendo-se às colheitas da cana-planta, soca e ressoca, respectivamente, nos dias 31 de agosto de 1988, 5 de setembro de 1989 e 31 de outubro de 1990, para os ensaios da Usina Costa Pinto, e nos dias 3 de agosto de 1988, 9 de agosto de 1989 e 15 de outubro de 1990, para os da Usina Santa Bárbara.

Por ocasião das colheitas, retiraram-se amostras de dez colmos seguidos na linha, em cada parcela, para análise do caldo. Também em multiplicação na Estação Experimental de Piracicaba, com três sulcos de $10 \mathrm{~m}$ para cada tratamento, espaçados de $1,40 \mathrm{~m}$, em latossolo vermelho-escuro orto, efetuaram-se amostragens mensais de abril a novembro de 1988 e 1989, na cana-planta e soca respectivamente, com duas amostras de dez canas seguidas por clone ou variedade para a determinação da curva de maturação, tendo por padrōes a 'NA56-79' e a 'CB41-76', como precoce e tardia repectivamente. As análises tecnológicas foram realizadas pelo método da prensa hidráulica (Tanimoto, 1964).

Realizaram-se as análises estatísticas individuais com as médias obtidas em cada experimento e, posteriormente, a análise conjunta para os dados de: produção de cana por área (t/ha); teor de açúcar (pol\% cana) e produção de álcool por área ( $\mathrm{t}$ de $\mathrm{pol} / \mathrm{ha}$ ). As médias de cada experimento e na análise conjunta foram comparadas pelo teste de Tukey a $5 \%$.

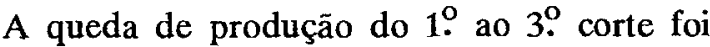
calculada com os dados de produção de cana (TCH), como se segue:

$$
\mathrm{Q} 3=\frac{3^{\circ} \text { corte }-1^{\circ} \text { corte }}{1^{\circ} \text { corte }} \times 100
$$

A análise estatística utilizou agrupamento por tratamentos comuns e as testemunhas comuns aos dois experimentos em cada local - 'NA56-79', 'CB41-76' e 'SP70-1143'. Para o caráter porcentagem de fibra, empregaram-se as médias conjuntas dos dois locais e três cortes na análise por agrupamento.

O florescimento foi avaliado por notas, correspondendo $0,1,2$ e 3 , respectivamente, a sem indução, apenas indução, 1 a $20 \%$ e 21 a $100 \%$ de florescimento. Os dados do teste de "carvão" foram fornecidos pela Comissão de Controle de Carvão da Cana-de-Açúcar do Estado de São Paulo $\left({ }^{9}\right)$.

(9) COMISSÃO DE CONTROLE DE CARVÃO DA CANA-DE-AÇÚCAR DO ESTADO DE SÃO PAULO, $14^{\circ}$ Teste oficial de carvâo. 1989. (Não publicado)

\section{RESULTADOS E DISCUSSÃO}

Os resultados médios de três cortes de produção de cana, pol\% cana e produção de açúcar por hectare, encontram-se nos quadros 1 e 2 .

Os clones de maior produtividade agrícola (TCH) foram o IAC74-107 e o IAC78-23, sendo que apenas o segundo se destacou da testemunha 'NA56-79' nos dois locais. Os clones IAC74-107, IAC78-13, IAC77-186 e IAC78-12 mostraram produtividades próximas às das melhores testemunhas 'SP70-1143' e 'IAC64-257', não diferindo estatisticamente delas na análise conjunta, e o clone IAC78-12 revelou produtividade estatisticamente maior em relação à 'NA56-79'. Os clones IAC78-54, IAC79-1011 e IAC79-1159 não diferiram estatisticamente de nenhuma das testemunhas para o caráter; entretanto, as produtividades foram semelhantes às das testemunhas 'NA56-79' e 'CB41-76', consideradas padrōes de produtividade média.

Analisando o teor de açúcar, expresso em pol\% cana, no ensaio das séries 74 e 77 da Usina Costa Pinto (Quadros 1 e 2), apenas o clone IAC74-107 foi significativamente inferior a todas as testemunhas.

Na Usina Santa Bárbara, o IAC74-107 não diferiu de nenhuma testemunha, fato esse um pouco conflitante ao obtido no outro local, sugerindo a necessidade de mais dados sobre o clone. A análise conjunta mostrou que o clone IAC74-107 não diferiu da 'CB41-76', porém foi estatisticamente menor que as demais testemunhas. Os clones IAC77-186, IAC74-353, IAC77-173, LAC77-51, IAC77-36, IAC77-153 e IAC74-339 não diferiram das testemunhas 'SP70-1143' e 'NA56-79' e foram significativamente superiores à 'CB41-76' na análise conjunta. $O$ clone IAC77-165 nāo diferiu estatisticamente de nenhuma testemunha.

No ensaio das séries 78 e 79 da Usina Santa Bárbara, os clones IAC78-90 e LAC79-1015 foram estatisticamente superiores a todas as testemunhas, exceto a 'NA56-79' na média dos três cortes. Já na Usina Costa Pinto, esses clones foram significativamente melhores em teor de açúcar à 'NA56-79' e 'CB41-76', porém nâo diferiram das testemunhas 'SP70-1143' e 'IAC64-257'. Na análise conjunta, foram estatisticamente superiores a todas as testemunhas. Para os clones IAC78-23, IAC79-1011 e IAC79-1159, as diferenças em relação às testemunhas 'LAC64-257' e 'SP70-1143' não foram significativas nos dois locais e na análise conjunta, sendo superiores à testemunha 'CB41-76' em quase todos os casos. Com 
relação à testemunha 'NA56-79', esses clones não diferiram em nenhum caso, exceto a média do IAC78-23 , que foi estatisticamente menor na Usina Santa Bárbara e comparativamente semelhante à 'CB41-76'.

Analisando os dados de produção de açúcar por hectare (TPH), na Usina Santa Bárbara, os clones IAC74-107 e IAC77-186 foram semelhantes estatisticamente às testemunhas $\mathrm{e}$, na Usina Costa Pinto, o clone IAC77-186 foi inferior apenas à 'SP70-1143'. $\mathrm{Na}$ análise conjunta dos dois clones, as médias foram menores que a da 'SP70-1143', em TPH. Por outro lado, na Usina Santa Bárbara, os clones IAC78-23, IAC78-90, IAC78-54, IAC79-1011, IAC79-1159 e IAC78-12 não diferiram estatisticamente das testemunhas do ensaio.

Na Usina Costa Pinto, os clones IAC78-23, IAC78-90, IAC79-1011 e IAC79-1159 superaram a 'CB41-76', e o LAC78-23 apresentou melhor média que a 'NA56-79'. Examinando a análise conjunta para o caráter, observa-se que os clones IAC78-23, IAC78-90 e LAC78-54 foram superiores à 'CB41-76', e o IAC78-23 foi superior à 'NA56-79'. Os clones IAC79-1011, IAC79-1159 e IAC78-12 não diferiram de nenhuma testemunha.

Por esses resultados, observa-se que os clones IAC74-107 e IAC78-12 apresentam boa produção, mas o teor de açúcar nâo é muito bom, e atualmente a exigência agroindustrial para o teor de açúcar é elevada. Na figura 1, e quadro 4, verifica-se que, na soqueira, o clone IAC74-107 tem bom teor de açúcar no início da safra, aumentando muito pouco depois no meio e no fim. O que se quer salientar é a necessidade de se testar este clone em outras regiões, para observar se se repete o teor de açúcar no início de safra.

No quadro 3 , observa-se também que o clone IAC78-12 mostra uma tendência de queda de produção mais acentuada que a 'IAC74-107', embora não haja diferenças estatísticas entre eles e com todas as testemunhas na Usina Costa Pinto. Na análise da Usina Santa Bárbara, a queda de produção no $3^{\circ}$ corte foi menor que no segundo, ultrapassando, em alguns casos, a produção do $1^{\circ}$ corte. Isso ocorreu em função de um período chuvoso mais intenso, após o $2^{\circ}$ corte até à colheita, do que nos anos anteriores.

Outro clone que se destacou foi o IAC77-186, com produção estatisticamente semelhante à da 'NA56-79' e queda de produção próxima à da 'IAC64 -257' : sua maturação é para meio e fim de safra (Figura 1 e Quadro 4), com altos valores de pol em outubro-novembro, podendo ser utilizada comercialmente nesse período, pois não apresentou florescimento, apenas indução. A produção de açúcar por hectare (TPH) foi equivalente às testemunhas 'NA56-79' e 'CB41-76', apesar de inferior à 'SP70-1143', caracterizando-o como material para fim de safra em solos de boa qualidade.

Quadro 1. Produção de cana, teor de açúcar e produção de açúcar por hectare (médias de três cortes) em dois locais e análises conjunta com clones das séries 74 e 77

\begin{tabular}{|c|c|c|c|c|c|c|c|c|c|}
\hline \multirow{2}{*}{ Tratamentos } & \multicolumn{3}{|c|}{ t/cana/ha (TCH) } & \multicolumn{3}{|c|}{ Pol \% cana (PC) } & \multicolumn{3}{|c|}{ t/pol/ha (TPH) } \\
\hline & Santa Bárbara & Costa Pinto & Média & Santa Bárbara & Costa Pinto & Média & Santa Bárbara & Costa Pinto & Média \\
\hline SP70-1143 & $154,4 a$ & $155,6 a$ & $155,0 \mathrm{a}$ & $14,70 \mathrm{ab}$ & $14,94 a$ & $14,82 a$ & $22,71 \mathrm{a}$ & $23,32 a$ & $23,01 a$ \\
\hline LAC74-107 & $147,0 \mathrm{ab}$ & $147,4 a$ & $147,2 \mathrm{a}$ & $14,22 \mathrm{ab}$ & $12,64 c$ & $13,43 \mathrm{~b}$ & $20,99 \mathrm{ab}$ & $20,03 a b$ & $20,51 b$ \\
\hline NA56-79 & $127,4 \mathrm{~cd}$ & $146,4 a$ & $136,9 \mathrm{~b}$ & $15,15 \mathrm{a}$ & $14,05 \mathrm{ab}$ & $14,60 \mathrm{a}$ & $19,24 b-d$ & $20,41 a b$ & $19,83 b$ \\
\hline CB41-76 & $151,5 \mathrm{ab}$ & $137,2 \mathrm{ab}$ & $144,3 a$ & $14,00 \mathrm{~b}$ & $12,84 b c$ & $13,42 b$ & $21,27 a b$ & $17,46 b-d$ & $19,37 \mathrm{bc}$ \\
\hline IAC77-186 & $133,8 b c$ & $135,2 \mathrm{ab}$ & $134,5 b$ & $14,79 \mathrm{ab}$ & $14,02 \mathrm{ab}$ & $14,41 a$ & $19,78 \mathrm{a}-\mathrm{c}$ & $18,63 b c$ & $19,21 b c$ \\
\hline IAC74-353 & $121,5 \mathrm{~cd}$ & $122,0 \mathrm{bc}$ & $121,7 \mathrm{bc}$ & $15,12 \mathrm{a}$ & $14,79 a$ & $14,96 \mathbf{a}$ & $18,34 b-d$ & $18,08 \mathrm{bc}$ & $18,21 b-d$ \\
\hline LAC77-173 & $117,0 \mathrm{~cd}$ & $124,1 \mathrm{bc}$ & $120,5 b c$ & $15,00 \mathrm{ab}$ & $14,15 a$ & $14,57 \mathbf{a}$ & $17,51 \mathrm{~cd}$ & $17,46 b-d$ & $17,49 \mathrm{~cd}$ \\
\hline IAC77-51 & $118,0 \mathrm{~cd}$ & $117,9 \mathrm{bc}$ & $117,9 \mathrm{c}$ & $14,85 a b$ & $14,74 a$ & $14,79 \mathbf{a}$ & $17,51 \mathrm{~cd}$ & $17,39 \mathrm{~b}-\mathrm{d}$ & $17,45 \mathrm{~cd}$ \\
\hline IAC77-36 & $109,7 \mathrm{de}$ & $130,7 a-c$ & $120,2 b c$ & $15,22 \mathrm{a}$ & $13,94 \mathbf{a b}$ & $14,58 \mathrm{a}$ & $16,39 \mathrm{de}$ & $18,19 b c$ & $17,29 \mathrm{~cd}$ \\
\hline IAC74-339 & $121,1 \mathrm{~cd}$ & $111,2 \mathrm{~cd}$ & $116,1 \mathrm{c}$ & $14,52 \mathrm{ab}$ & $14,68 \mathrm{a}$ & $14,60 \mathrm{a}$ & $17,75 \mathrm{~cd}$ & $16,34 \mathrm{~cd}$ & $17,05 \mathrm{~cd}$ \\
\hline IAC77-165 & $116,4 \mathrm{~cd}$ & $117,2 b c$ & $116,8 c$ & $14,54 a b$ & $13,86 a b$ & $14,20 \mathrm{ab}$ & $17,05 \mathrm{c}-\mathrm{e}$ & $16,39 \mathrm{~cd}$ & $16,72 d$ \\
\hline IAC77-153 & $96,1 \mathrm{e}$ & $95,5 \mathrm{~d}$ & $95,8 \mathrm{~d}$ & $14,69 \mathrm{ab}$ & $14,99 \mathrm{a}$ & $14,84 a$ & $14,14 \mathrm{e}$ & $14,34 d$ & $14,24 \mathrm{e}$ \\
\hline Valor de "F" & $19,76^{* *}$ & $12,33^{* *}$ & $26,11^{* *}$ & $2,76^{*}$ & $8,60^{* *}$ & $8,24^{* *}$ & $12,59^{* *}$ & $10,29^{* *}$ & $19,88^{* *}$ \\
\hline d.m.s. (Tukey a 5\%) & 18,7 & 20,6 & 14,4 & 1,04 & 1,21 & 0,83 & 3,17 & 3,31 & 2,33 \\
\hline CV\% & 12,15 & 13,42 & 13,27 & 3,90 & 7,15 & 6,78 & 13,96 & 15,25 & 14,85 \\
\hline
\end{tabular}


CANA PLANTA, 1988
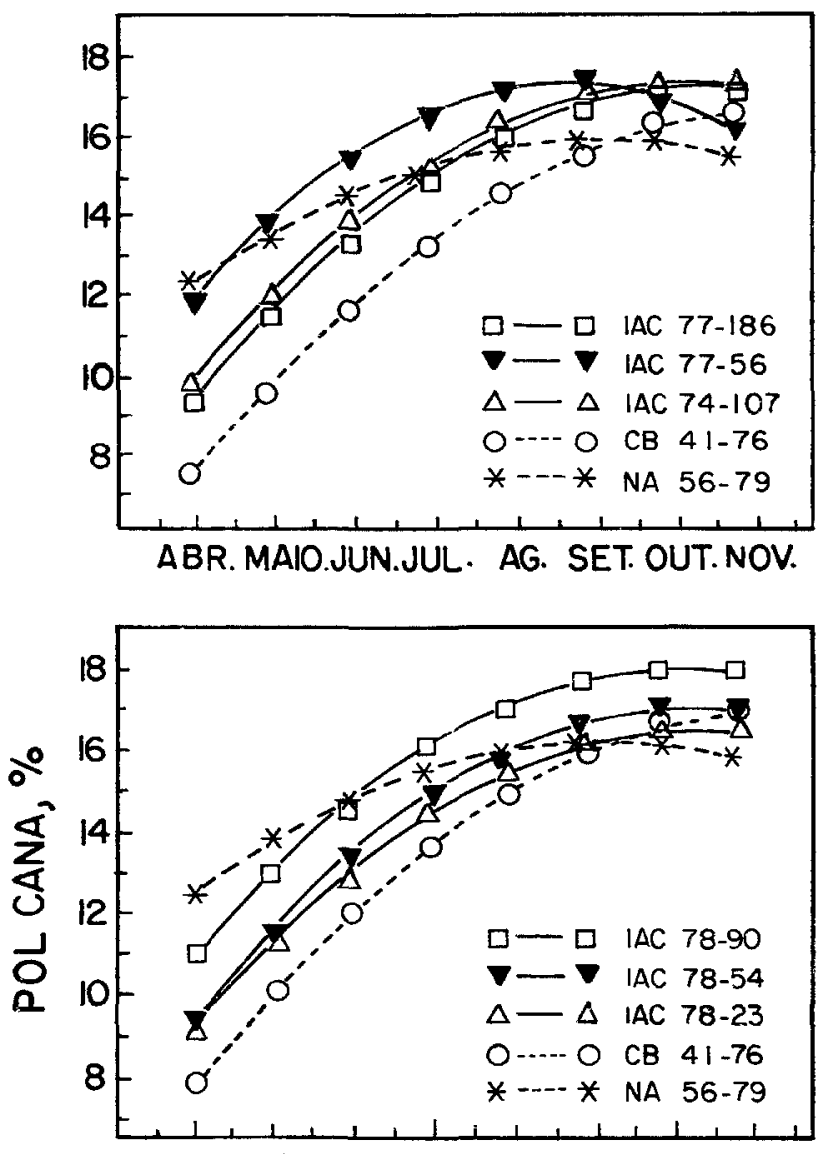

ABR. MAIO JUN JUL. AG. SET. OUT.NOV.

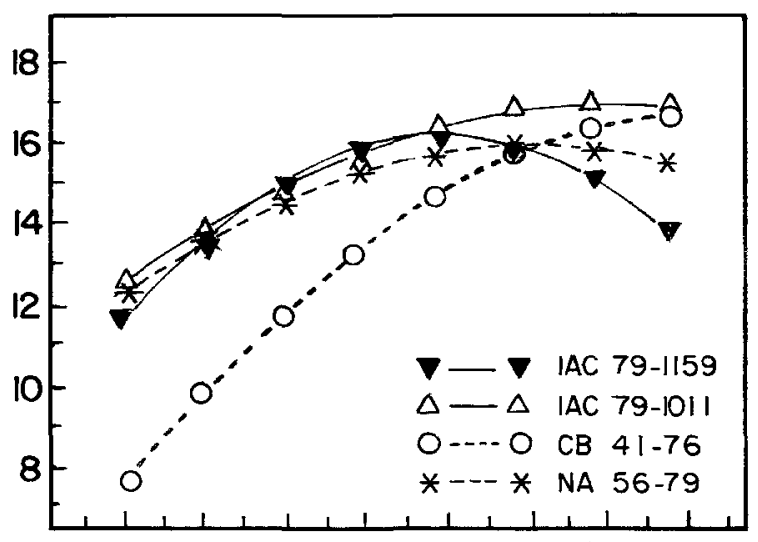

ABR. MAIO JUN.JUL. AG. SET. OUT. NOV.
CANA SOCA, 1989
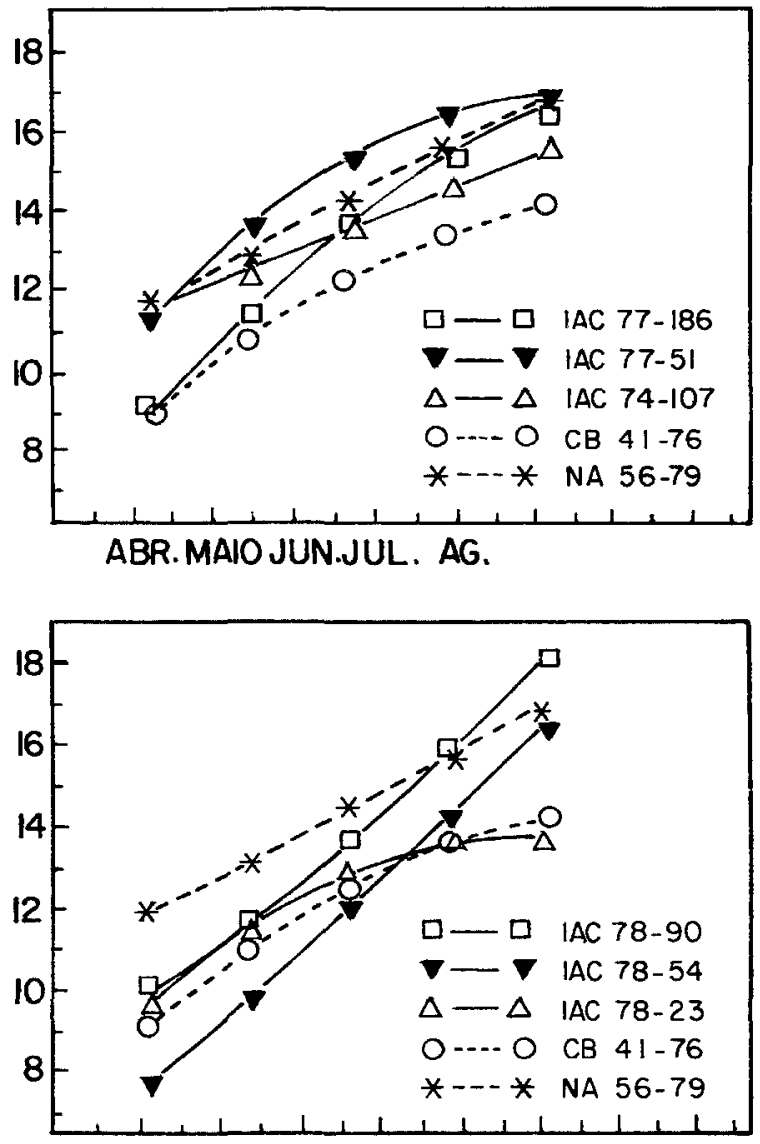

ABR. MAIO JUN.JUL. AG.

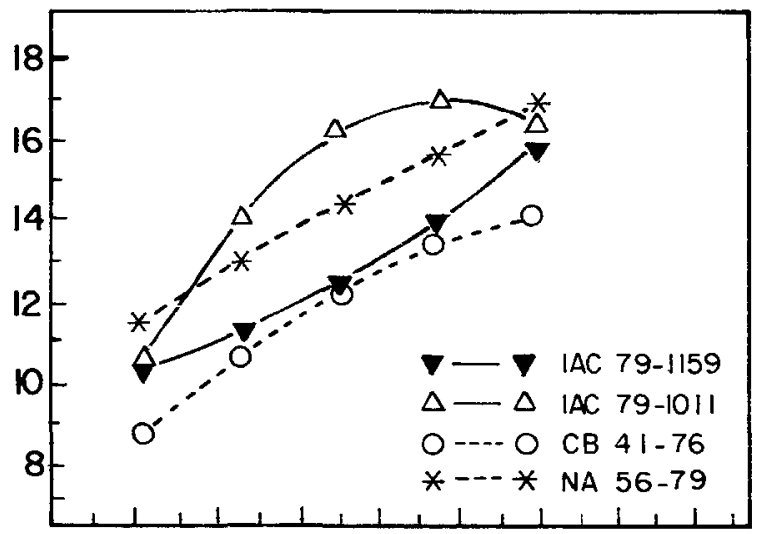

ABR. MAN JUN.JUL. AG.

\section{MESES DO ANO}

Figura 1. Curvas de maturação de clones IAC e testemunhas na cana-planta e soca, em ensaio realizado na Estação Experimental de Piracicaba (SP). 
Quadro 2. Produção de cana, teor de açúcar e produção de acúcar por hectare (médias de três cortes) em dois locais e análise conjunta com clones das séries 78 e 79

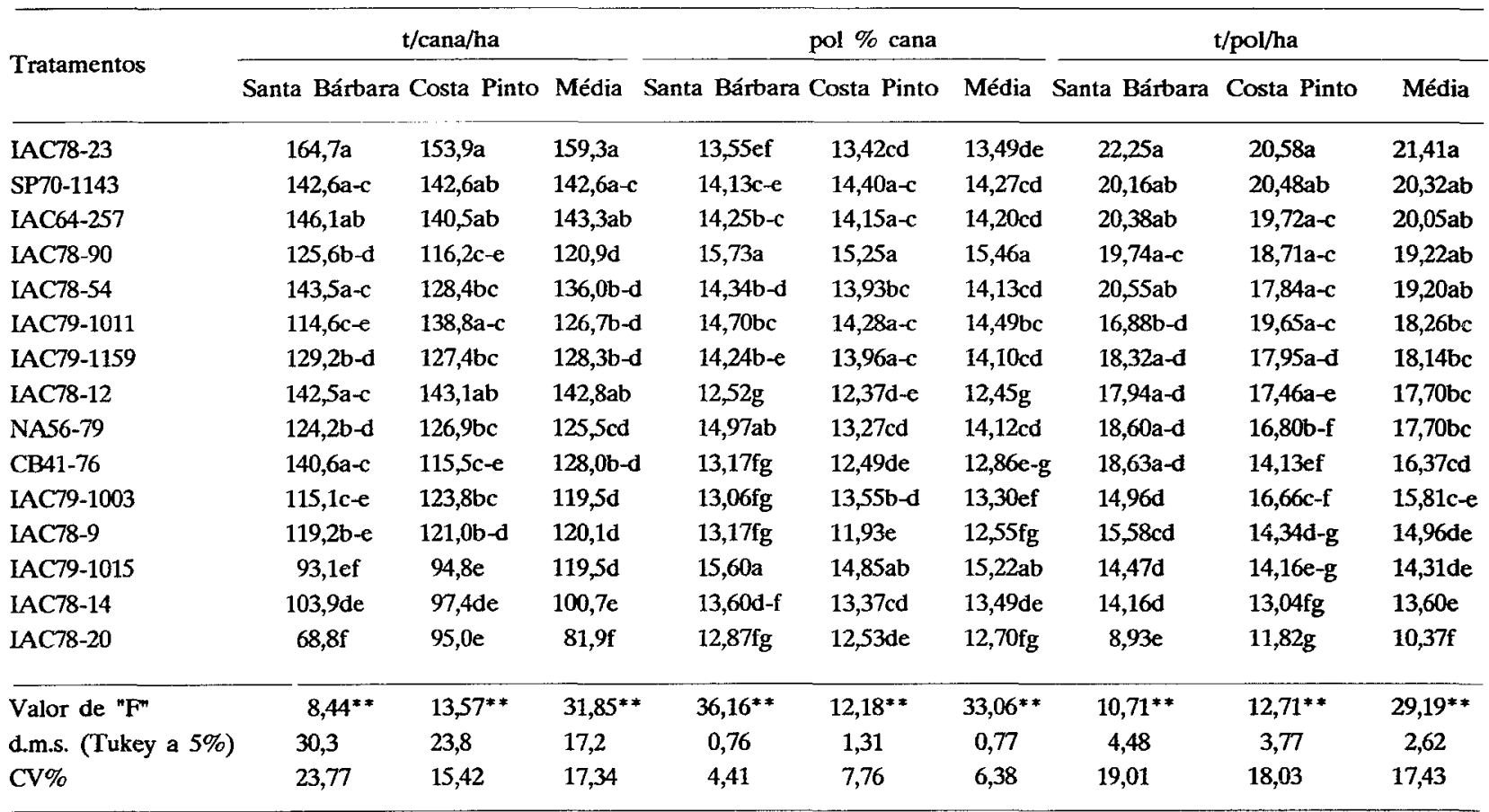

O clone LAC78-23 é de alta produçāo agrícola, superior às testemunhas 'NA56-79' e 'CB41-76', com queda de produção (Q3) (Quadro 3) semelhante à testemunha 'IAC64-257', o que caracteriza o material como de baixa exigência. No teste oficial de carvão, foi caracterizado como suscetível; entretanto, ao nível de ensaio, a infestação foi nula, enquanto a testemunha 'NA56-79' apresentava $30 \%$ de infestação.

Em vista disso, recomenda-se o plantio restrito a $10 \%$ da área, para náo aumentar o potencial de inóculo na lavoura. A maturação é média a tardia e não floresce, podendo, entāo, ser colhida em meio e fim de safra. A produção de açúcar por área supera as testemunhas 'NA56-79' e 'CB41-76', mostrando alto rendimento agroindustrial.

Com produção média a alta e queda de produção próxima à da 'IAC64-257', o clone IAC78-54 também possui maturação média a tardia. Como floresce um pouco, seu período de industrialização pode ser curto, restringindo-se a meio de safra. Possui reaçāo intermediária ao carvão, o que náo o recomenda para plantios em maiores porcentagens que $15 \%$ da área. A produção de açúcar por área é intermediária entre a da 'SP70-1143' e 'NA56-79', sem, no entanto, diferir estatisticamente, caracterizando o material como de certa exigência em fertilidade.

Já para o clone IAC78-90, a produção esteve próxima à da 'CB41-76' e 'NA56-79', e queda de produção no $3^{\circ}$ ' corte semelhante à da 'CB41-76', que possui uma queda mais acentuada entre as testemunhas, o que coloca o clone para uso em solos férteis. É um material com altos valores de açúcar em meio e fim de safra, sendo na soqueira um pouco mais tardio. Floresce um pouco e, portanto, deve ser colhido em meio de safra na cana-planta e fim de safra na soca, onde ocorre menos florescimento. Para o IAC78-54, a produção de açúcar por área é intermediária com relação às testemunhas 'SP70-1143' e ‘CB41-76’; além disso, é suscetível ao carvão em testes inoculados, mas não mostrou infestação nos ensaios, onde a infestação da 'NA56-79' era de $30 \%$. Em função dessas considerações, também pode ser utilizada sem ocupar grandes extensōes de canavial, o que pressionaria a ocorrência de infestação, como no caso do IAC78-23. 
Quadro 3. Dados de queda de produção da $1^{\text {a à }} 3^{\text {a }}$ colheita, de reação ao "carvão", fibra e florescimento

\begin{tabular}{|c|c|c|c|c|c|}
\hline \multirow{2}{*}{ Tratamentos } & \multicolumn{2}{|c|}{ Queda de produçāo $\left({ }^{1}\right)$} & \multirow{2}{*}{$\begin{array}{c}\text { Reação } \\
\left.\text { ao "carvão" }{ }^{2}\right)\end{array}$} & \multirow{2}{*}{ Fibra } & \multirow{2}{*}{ Florescimento } \\
\hline & Costa Pinto & Santa Bárbara & & & \\
\hline IAC74-107 & $-32,67 \mathrm{~b}-\mathrm{e}$ & $+9,05 a-c$ & $\mathbf{R}$ & $12,58 \mathrm{a}-\mathrm{c}$ & 2 \\
\hline LAC74-339 & $-49,49 a-c$ & $-6,98 a-c$ & $\mathbf{R}$ & $11,68 a-c$ & 2 \\
\hline LAC74-353 & $-51,60 \mathrm{ab}$ & $-21,17 \mathbf{a}$ & $\mathbf{R}$ & $11,30 \mathrm{a}-\mathrm{c}$ & 2 \\
\hline LAC77-36 & $-29,50 b-f$ & $+9,35 \mathrm{a}-\mathrm{c}$ & $\mathbf{R}$ & $12,60 a-c$ & 0 \\
\hline IAC77-51 & $-41,29 a-d$ & $+3,26 a-c$ & $\mathbf{R}$ & $11,97 a-c$ & 0 \\
\hline LAC77-153 & $-7,42 \mathrm{e}-\mathrm{f}$ & $+9,66 \mathrm{a}-\mathrm{c}$ & $\mathbf{S}$ & $12,54 a-c$ & 2 \\
\hline IAC77-165 & $-39,60 a-d$ & $-14,17 a-b$ & $\mathbf{R}$ & $12,09 a-c$ & 3 \\
\hline IAC77-173 & $-25,75 c-f$ & $-3,70 a-c$ & $\mathbf{R}$ & $12,10 \mathrm{a}-\mathrm{c}$ & 1 \\
\hline LAC77-186 & $-31,94 b-e$ & $+4,48 \mathrm{a}-\mathrm{c}$ & $\mathbf{R}$ & $11,89 \mathrm{a}-\mathrm{c}$ & 1 \\
\hline IAC78-9 & $-16,05 d-f$ & $+4,89 \mathrm{a}-\mathrm{c}$ & $\mathbf{R}$ & $11,97 a-c$ & 2 \\
\hline IAC78-12 & $-48,21 a-c$ & $-7,60 a-c$ & $\mathbf{R}$ & $10,36 \mathrm{c}$ & 1 \\
\hline LAC78-14 & $-40,87 a-d$ & $-9,47 a-c$ & $\mathbf{R}$ & $12,60 \mathrm{a}-\mathrm{c}$ & 0 \\
\hline IAC78-20 & $-61,03 a$ & $-21,31 a$ & $\mathbf{S}$ & $11,12 b$ & 1 \\
\hline IAC78-23 & $-34,65 b-e$ & $+14,45 \mathrm{bc}$ & $\mathbf{S}$ & $12,57 \mathrm{a}-\mathrm{c}$ & 0 \\
\hline LAC78-54 & $-33,67 b-d$ & $-5,11 a-c$ & I & $13,56 \mathrm{a}$ & 2 \\
\hline LAC78-90 & $-44,90 a-c$ & $-18,53 a$ & $\mathbf{S}$ & $11,37 \mathrm{a}-\mathrm{c}$ & 2 \\
\hline IAC79-1003 & $+4,95 \mathrm{f}$ & $+10,68 \mathrm{a}-\mathrm{c}$ & $\mathbf{R}$ & $12,26 a-c$ & 2 \\
\hline IAC79-1011 & $-29,93 b-f$ & $-13,51 \mathrm{ab}$ & I & $11,72 \mathrm{a}-\mathrm{c}$ & 2 \\
\hline LAC79-1015 & $-32,29 b-e$ & $+20,78 c$ & I & $13,29 a-b$ & 3 \\
\hline LAC79-1159 & $-36,28 a-d$ & $0,0 a-c$ & I & $12,03 a-c$ & 2 \\
\hline NA56-79 & $-30,64 b-e$ & $+15,48 b c$ & $\mathrm{~S}$ & $11,82 a-c$ & 1 \\
\hline CB41-76 & $-47,47 a-c$ & $+6,37 \mathrm{a}-\mathrm{c}$ & $\mathbf{R}$ & $10,81 \mathrm{c}$ & 0 \\
\hline SP70-1143 & $-29,28 b-e$ & $+4,19 a-c$ & $\mathbf{R}$ & $12,32 a-c$ & 3 \\
\hline IAC4-257 & $-34,60 b-d$ & $+1,75 a-c$ & $\mathbf{R}$ & $13,27 a-b$ & 2 \\
\hline Valor de $\mathrm{F}$ & $15,24^{* *}$ & $11,25^{* *}$ & & $6,91^{* *}$ & \\
\hline d.m.s. (Tukey a 5\%) & 25,60 & 32,22 & & 2,30 & \\
\hline CV\% & 24,65 & 31,17 & & 9,06 & \\
\hline
\end{tabular}

( $\left.{ }^{1}\right)$ Negativo para queda de produção e positivo para aumento. $\left(^{2}\right) \mathrm{R}=$ resistente; $\mathrm{S}=$ suscetível; $\mathrm{I}=$ intermediário.

O clone IAC79-1011 também é comparável em produção à 'CB41-76' e 'NA56-79'; apenas sua queda de produção é pequena, como na 'SP70-1143' e 'NA56-79'; portanto, qualitativamente menos exigente em tipos de solos que a 'CB41-76'. Além disso, possui bons teores de açúcar e é precoce na maturação, indicada para manejo em início de safra, onde é raro encontrar opcooes de cultivo. Todavia, em função da produção agrícola, a produção de açúcar por área fica próxima da 'NA56-79' e 'CB41-76', devendo ser explorada em solos mais férteis para obter maiores rendimentos. Sua reação ao carvão é intermediária, devendo-se evitar, assim, áreas extensas deste clone.

Tudo o que foi dito para produção, teores de açúcar e produção de açúcar por área, do clone LAC79-1011, pode-se aplicar ao IAC79-1159, apenas que a 
Quadro 4. Fórmulas de regressão quadrática e valores de coeficiente de determinação das curvas de maturaçāo dos clones promissores

Clone/Variedade

Estádio

Fórmula

$\mathbf{R}^{2}$

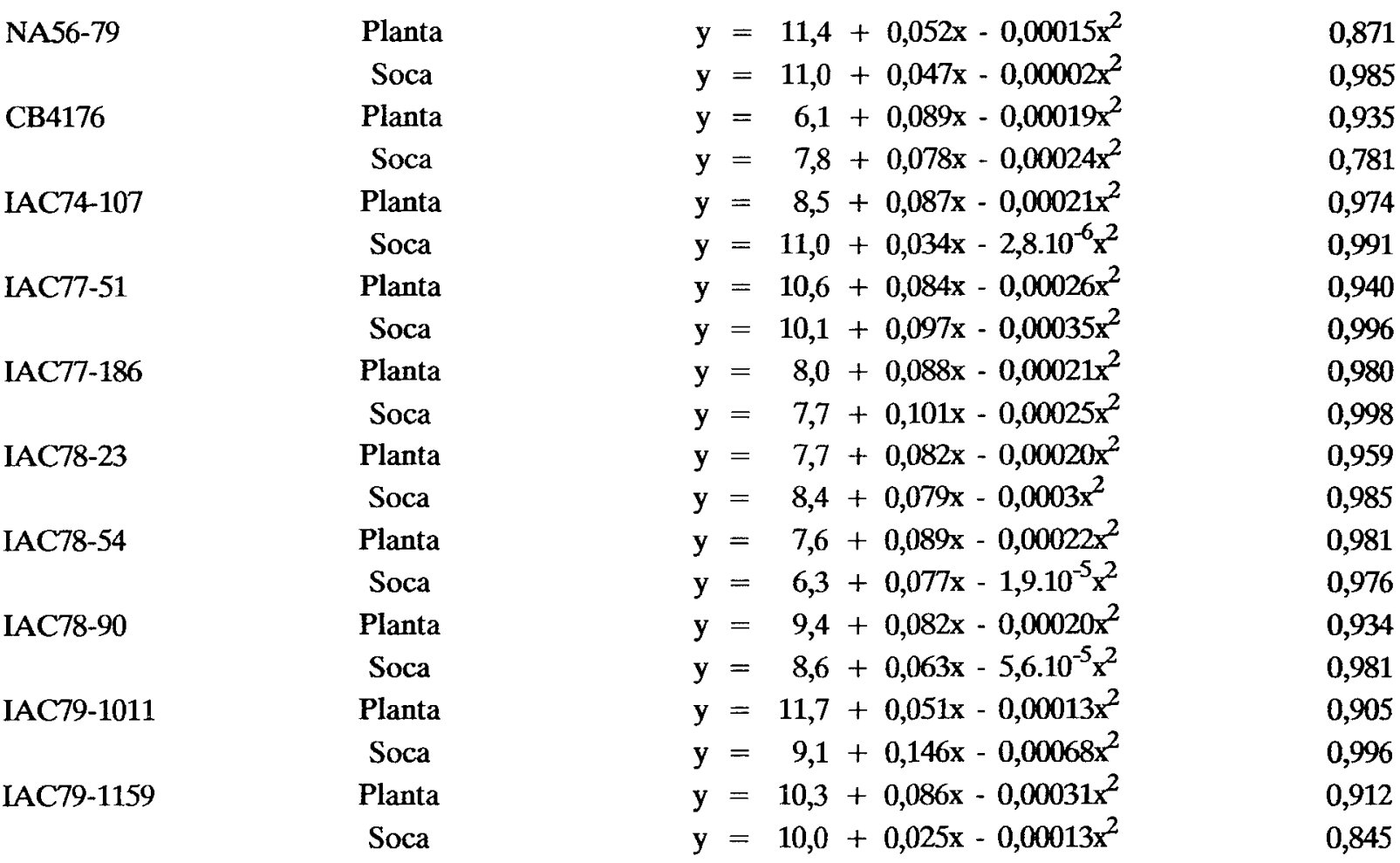

maturação é precoce na cana-planta, média a tardia na soca; como floresce um pouco, recomenda-se o corte em início e meio de safra na planta, e meio e fim na soca, onde o florescimento é menos intenso. Também possui reação intermediária ao carvão.

Os demais clones do ensaio possuem muitas limitaçōes agroindustriais, não sendo recomendados para cultivo. No entanto, o IAC77-51 deve ser indicado para o banco de germoplasma em função do seu alto teor de açúcar, para utilização em futuras hibridações. O clone IAC74-107 deve ser retestado para teor de açúcar.

\section{CONCLUSŌES}

Podem ser estabelecidos, como opção de cultivo para a regiāo de Piracicaba, os seguintes clones:
- IAC77-186: como material exigente em solos de alta fertilidade e de maturaçāo tardia;

- IAC78-23: material produtivo, não exigente para colheita em meio e fim de safra, com restrições ao uso extensivo; de safra;

- IAC78-54: produção média, restrito ao meio

- IAC78-90: produção média, uso para meio e fim de safra e muito exigente, tem restrições para uso extensivo;

- IAC79-1011: produção média, exigente, indicada para uso restrito em início de safra;

- IAC79-1159: produção média, pouco exigente, com uso no início e meio na planta e meio e fim na soca. 


\section{AGRADECIMENTOS}

À COPERSUCAR, pela possibilidade de realizar os cruzamentos; à Usina Santa Bárbara e seus engenheiros João Américo Beltrame e Marco Aurélio Sansigolo, como também à Usina Costa Pinto e seus engenheiros Renato Soffener e Plínio Lara Neto, pelo apoio na condução dos ensaios.

\section{REFERENCIAS BIBLIOGRÁFICAS}

AGUIRRE JUNIOR, J.M. de. Criaçáo de novas variedades de canna no Estado de São Paulo. Campinas, Instituto Agronômico, 1936. 64p. (Boletim technico. 34)

ALVAREZ, R; BASTOS, C.R; LANDELL, M.G. de A.; BOVI, V.; POMMER, C.V.; PEREIRA, J.C.V.N.A.; SILVAROLLA, M.B.; GODOY JÚNIOR, G.; COSTA, A.A.; CAMARGO, A.P. de \& RAMOS, M.T.B. Melhoramento da cana-de-açúcar: V. Experimentos regionais com clones obtidos em 1970. STAB, Piracicaba, 4(4):39-46, 1986.

ALVAREZ, R; BASTOS, C.R.; LANDELL, M.G. de A.; BOVI, V.; POMMER, C.V.; PEREIRA. J.C.V.N.A.; SILVAROLLA, M.B.; GODOY JUNIOR, G.; COSTA, A.A.; CAMARGO, A.P. de \& RAMOS, M.T.B. Cana-de-açúcar 'IAC70-22'. O Agronômico, Campinas, 39(2):107-108, 1987a.

ALVAREZ, R; BASTOS, C.R.; POMMER, C.V.; BRINHOLI, O.; DALBEN, A.E.; GODOY JUNIOR, G.: BOVI, V. \& CIONE, J. Melhoramento da cana-de-açúcar. III. Experimentos regionais com clones obtidos em 1968. In: CONGRESSO NACIONAL DA SOCIEDADE DE TÉCNICOS AÇUCAREIROS DO BRASIL, 2., e SIMPÓSIO LATINO-AMERICANO SOBRE MODALIDADES DE FINANCIAMENTO À PRODUÇĀO DE ENERGIA RENOVÁVEL, 1., Rio de Janeiro, 1981. Anais. Rio de Janeiro, STAB, 1981. v.2, p.198-215.
ALVAREZ, R.; BASTOS, C.R; SEGALLA, A.L;; OLIVEIRA, H. de; GODOY JUNIOR, G.; POMMER, C.V.; BRINHOLI, O. \& DALBEN, A.E. Melhoramento da cana-de-açúcar. IIa. Experimentos regionais com clones em 1967. Bragantia, Campinas, 42:27-36, 1983.

ALVAREZ, R.; POMMER, C.V.; BASTOS, C.R.; BRINHOLI. O.; GODOY JUNIOR, G. \& BOVI, V. Melhoramento da cana-de-açúcar. IV. Experimentos regionais com clones obtidos em 1969. Bragantia, Campinas, 43(1):155-165, 1984.

ALVAREZ, R.; SEGALLA, A.L.; LANDELL, M.G. de A.; SILVAROLLA, M.B. \& GODOY JUNIOR, G. Melhoramento genético da cana-de-açúcar: avaliação de clones provenientes de hibridações efetuadas em 1965. Bragantia, Campinas, 46(1):121-126, $198 \%$.

BRIEGER, F. Situação do melhoramento da cana-de-açúcar no Estado de São Paulo. In: S0 ANOS da Estação Experimental de Piracicaba. Campinas, Instituto Agronômico, 1978. p.13-18.

CAMPANA, M.P.; SILVAROLLA, M.B.; LANDELL, M.G. de A. \& ALVAREZ, R. Comportamento agroindustrial de clones IAC de cana-de-açúcar, série 1980 em latossolo vermelho-escuro. STAB, Piracicaba, 8(5/6):22-25, 1990.

MACHADO JUNIOR, G.R.; MENDONÇA, J.R. de \& SILVA, J.A. da. Censo varietal quantitativo 1987. Boletim Técnico Copersucar, São Paulo, (4Ș):10, 1989.

SEGALlA, A.I. \& ALVAREZ, R. Melhoramento da cana-de-açúcar: I. Experiências com os "seedlings" obtidos em 1947, 1948 e 1949. Bragantia, Campinas, 23:187-223, 1964.

SEGALLA, A.L.; ALVAREZ, R.; OLIVEIRA, H. de; IGUE, T. \& GODOY JUNIOR, G. Melhoramento da cana-de-açúcar: II. Experimentos regionais com clones obtidos em 1964. Bragantia, Campinas, 41:109-117, 1982.

SEGALLA, A.L.; OLIVEIRA, H. de; FSPIRONELO, A. \& IGUE, T. Experimentos regionais de variedades de cana-de-açúcar realizados no período de 1970 a 1973. Bragantia, Campinas, 39:115-135, 1980.

TANIMOTO, T. The press method of cane analysis. Hawaiian Planter's Record, Honolulu, 57(2):133-150, 1964. 